\title{
Efficacy and comparative uptake rates of sublingual and capsular vitamin D preparations
}

\author{
C.E. Williams ${ }^{1}$, E.A. Williams ${ }^{2}$ and B.M. Corfe $\mathrm{e}^{1,3}$ \\ ${ }^{1}$ Molecular Gastroenterology Research Group, Academic Unit of Surgical Oncology, ${ }^{2}$ Human Nutrition Unit, \\ Department of Oncology \& Metabolism, University of Sheffield, Beech Hill Road, Sheffield, S10 2 RX and ${ }^{3}$ Insigneo \\ Institute for In Silico Medicine, The University of Sheffield.
}

Vitamin D is critical for skeletal health and is increasingly associated with other pathologies encompassing gastrointestinal, immunological, psychological effects ${ }^{(1)}$. A significant proportion of the population exhibit suboptimal levels of vitamin D, particularly in Northern latitudes in winter ${ }^{(2)}$. Supplementation is advocated, but few data are available on relative efficacy of preparations, or rates of uptake, or whether serum status may influence uptake. Sublingual sprays are an emerging platform for delivery of nutrient supplements, but data on efficacy remains sparse. A randomised, placebo-controlled, 3-arm parallel design study was conducted in healthy volunteers $(n=75)$ to compare uptake rates of vitamin $\mathrm{D}$ supplementation in capsule and sublingual spray preparations over a six-week period between January and April 2017. Serum 25(OH)D concentrations were measured after day 0, 3, 7, 14, 21 and 42 days of supplementation with 3000IU per diem. Baseline measurements show 25(OH)D deficiency, insufficiency and sufficiency in $14.9 \%, 44.6 \%$ and $40.5 \%$ of the participants respectively. There was a significant elevation in serum concentrations of $25(\mathrm{OH}) \mathrm{D}$ in the treatment arms (capsule $p=0.003$, spray $p=0.001)($ Fig. $1 \mathrm{~A})$ compared to control. The capsule and spray were equally efficacious with average change in serum vitamin $\mathrm{D}$ of $2 \mathrm{nmol} / \mathrm{ml} /$ day. Correlations between uptake rate and baseline serum vitamin $\mathrm{D}$ data suggest that uptake rates are higher in individuals with lower serum vitamin D (Fig. 1B + C) irrespective of delivery platform.

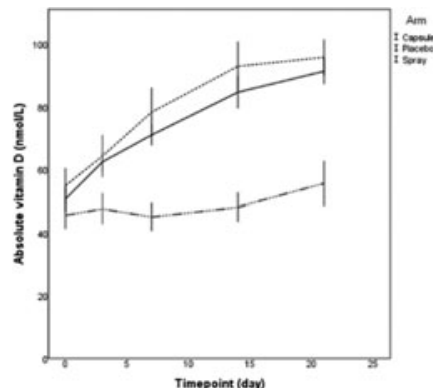

Fig. 1A. Increase in vitamin D over 3 weeks. Grey line - capsules; black line spray, dashed line, placebo.

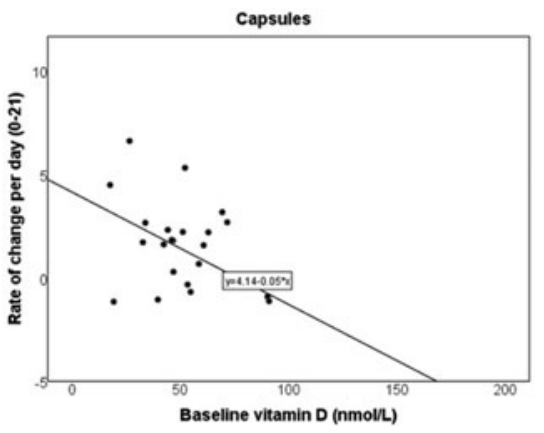

Fig. 1B. Rate of change in vitamin $\mathrm{D}(\mathrm{nmol} / \mathrm{ml} /$ day) versus baseline serum vitamin $\mathrm{D}(\mathrm{nmol} / \mathrm{l})$ in capsule arm.

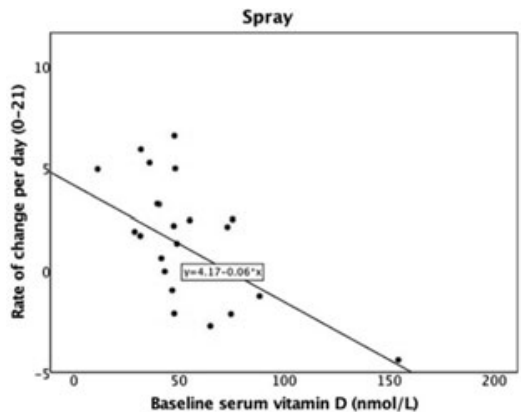

Fig. 1C. Rate of change in vitamin $\mathrm{D}(\mathrm{nmol} / \mathrm{ml} /$ day) versus baseline serum vitamin $\mathrm{D}(\mathrm{nmol} / \mathrm{l})$ in spray arm.

In conclusion, a sublingual vitamin D spray is an effective and preferential mode of delivery for supplementation in a healthy population. Achievable rates of vitamin D increment are suggested to be around $2 \mathrm{nmol} / \mathrm{ml} / \mathrm{day}$. The data suggest a possible homeostatic mechanism for vitamin D uptake.

1. Ross AC, Manson JE, Abrams SA, Aloia JF, Brannon PM, Clinton SK, et al. (2011) The 2011 Report on Dietary Reference Intakes for Calcium and Vitamin D From the Institute of Medicine: What Clinicians Need to Know. Obstetrical \& Gynecological Survey 66(6), 356-7.

2. Lanham-New SA \& Wilson LR (2016) Vitamin D - has the new dawn for dietary recommendations arrived? Journal of Human Nutrition and Dietetics 29(1), 3-6. 